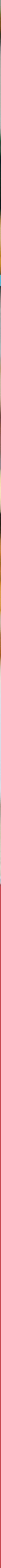




\section{Comment les enseignants maintiennent-ils leurs compétences pédagogiques à jour face à un monde en rapide mutation?}

- Selon l'Enquête internationale sur l'enseignement et l'apprentissage (TALIS), la qualité du développement professionnel proposé aux enseignants et le type d'activités s'y rapportant sont liés à plusieurs aspects essentiels de la profession enseignante, tels que la satisfaction au travail et l'efficacité personnelle.

- En moyenne, dans les pays et les économies de l'OCDE participant à l'Enquête TALIS, trois enseignants du premier cycle du secondaire sur quatre ont suivi des cours ou des séminaires au cours de l'année précédant l'Enquête.

- Les enseignants du premier cycle du secondaire ont répondu qu'ils avaient un besoin important en matière de développement professionnel dans le domaine de "l'enseignement aux élèves ayant des besoins spécifiques d'éducation », " des compétences en TIC à l'appui de l'enseignement » et de « l'enseignement en milieu multiculturel ou plurilingue ».

- Plus de $50 \%$ des enseignants dans près de la moitié des pays et des économies participant à l'Enquête TALIS reconnaissent que leur participation aux activités de développement professionnel est entravée par des incompatibilités avec leur emploi du temps ou par l'absence d'incitation.

\section{La participation à des activités de développement professionnel est répandue chez les enseignants}

La participation des enseignants à des activités de développement professionnel continu (DPC) leur permet de développer et de mettre à jour leurs compétences, leurs connaissances et leur expertise au profit de l'apprentissage des élèves. Cette participation, qui complète les programmes de formation initiale et d'initiation, est presque universelle dans les pays de l'OCDE et constitue un élément fondateur du professionnalisme des enseignants.

Selon l'Enquête internationale sur l'enseignement et l'apprentissage (TALIS) de l'OCDE, en moyenne, dans les pays et les économies de l'OCDE participant à l'Enquête TALIS, 94 \% des enseignants du premier cycle du secondaire indiquent qu'ils ont participé au moins une fois à une activité de développement professionnel continu au cours de l'année précédant l'Enquête. Ce taux de participation dépasse 90 \% dans la plupart des pays et reste au-dessus de 80 \% en Arabie saoudite, au Brésil, au Chili, en France et au Portugal.

Les activités de développement professionnel prennent diverses formes (voir le graphique 1). Les formes d'activités les plus répandues sont les « cours/séminaires en présentiel » (76 \%) et la « lecture d’ouvrages spécialisés » (72 \%), suivies par les « conférences pédagogiques » (49\%) et la formation basée sur l'apprentissage et l'accompagnement par les pairs (44 \%).

\section{Qu'est-ce que l'Enquête TALIS ?}

Lancée en 2008, l'Enquête internationale sur l'enseignement et l'apprentissage (TALIS) est la première grande étude internationale auprès d'enseignants et de chefs d'établissement qui examine différents aspects ayant une incidence sur l'apprentissage des élèves. Elle donne la parole aux enseignants et aux chefs d'établissement, leur permettant de contribuer à l'analyse et au développement des politiques en éducation dans des domaines clés. La population internationale visée par l'Enquête TALIS est composée des enseignants et des chefs d'établissement du premier cycle du secondaire dans les principaux établissements publics et privés. Dans chaque pays, un échantillon représentatif de 4000 enseignants et de leurs chefs dans 200 établissements a été sélectionné de manière aléatoire pour l'étude 2018. Environ 260000 enseignants ont fourni des réponses en lien avec toutes les composantes de l'Enquête, ce qui représente plus de 8 millions d'enseignants de 48 pays et économies participant à l'Enquête.

Pour plus d'informations, veuillez consulter le site suivant : www.oecd.org/education/talis.

\section{Le développement professionnel a des retombées majeures sur les pratiques pédagogiques}

En moyenne, dans les pays et les économies de l'OCDE participant à l'Enquête TALIS, quatre enseignants sur cinq (82 \%) indiquent que le développement professionnel a des retombées positives sur leurs pratiques pédagogiques (OCDE, Base de données TALIS 2018, tableau I.5.15). Les bénéfices perçus atteignent des niveaux particulièrement élevés en Alberta (Canada), en Australie, dans la Ciudad Autónoma de Buenos Aires (CABA, Argentine), au Japon et à Singapour, où au moins $90 \%$ des enseignants font état de retombées positives. En revanche, en Belgique, en Bulgarie, au Danemark, en France, à Malte, en 


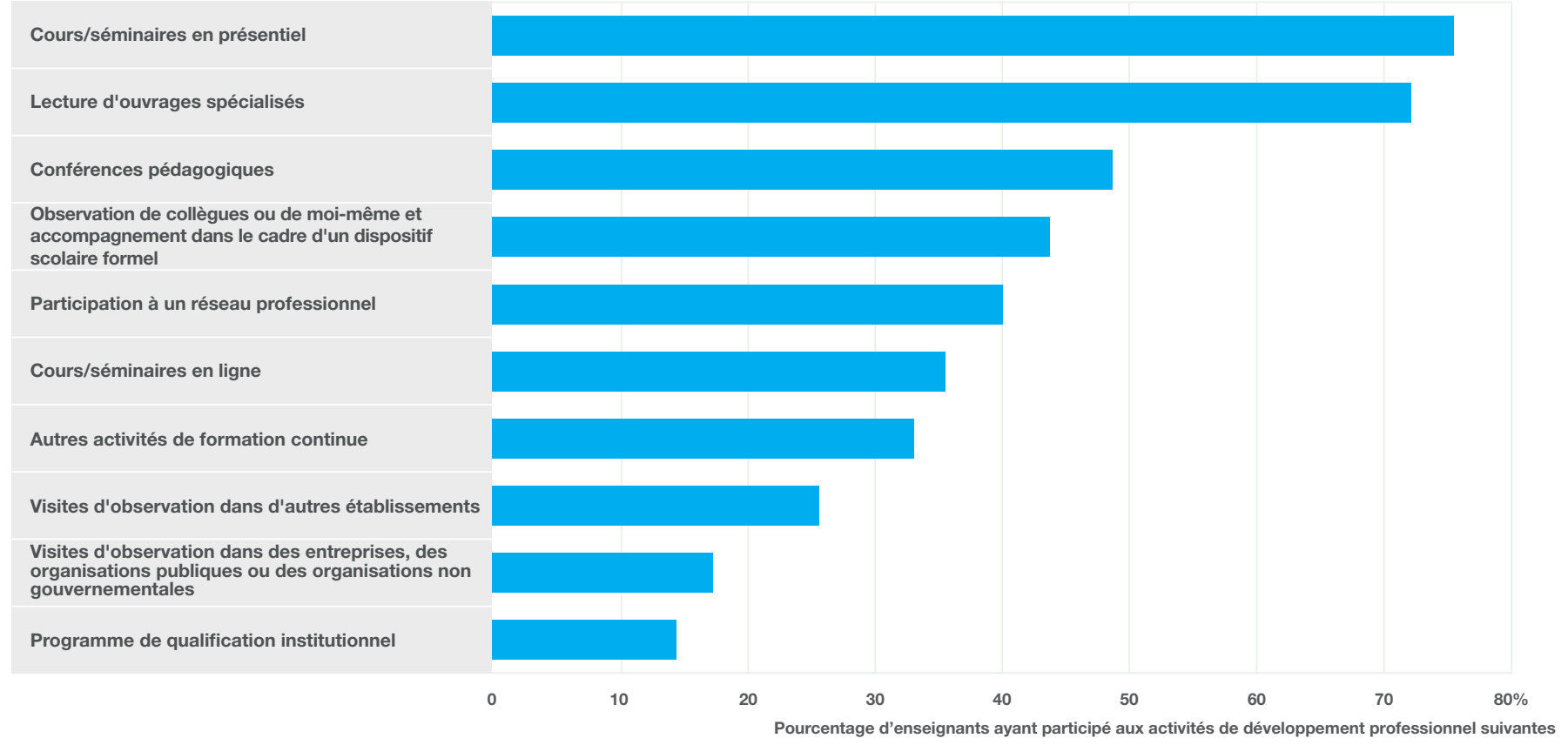

Remarque : La moyenne de l'OCDE couvre 31 pays (voir OCDE, 2019, Annexe B). Ce graphique répertorie les activités de développement professionnel auxquelles les enseignants ont participé au cours des douze mois précédant l'Enquête.

Source : OCDE, Base de données TALIS 2018, tableau 1.5.7.

Suède et en Turquie, seuls près de 70 \% des enseignants semblent estimer que leurs activités de développement professionnel sont constructives.

En général, les enseignants indiquent que les activités de développement professionnel les plus efficaces « sont construites sur les connaissances préalables de l'enseignant » (91 \% en moyenne dans les pays et les économies de l'OCDE participant à l'Enquête TALIS) et " prévoient des possibilités de mettre en pratique/d'appliquer de nouveaux concepts et savoirs dans la salle de classe » (86\%). Les activités qui mettent l'accent sur les contenus à enseigner tout en y associant des possibilités de collaboration et d'apprentissage actif semblent être les types d'activités les plus efficaces dans le domaine du DPC.

La participation à des activités de développement professionnel efficaces présente une corrélation positive avec différentes dimensions affectant la profession enseignante, telles que la satisfaction professionnelle des enseignants (observée dans 46 des 48 pays et économies de l'Enquête TALIS) et l'efficacité personnelle (observée dans 33 des 48 pays et économies de l'enquête TALIS) (OCDE, Base de données TALIS 2018, tableaux I.5.13 et I.5.14).

\section{Le nombre insuffisant d'activités pertinentes de développement professionnel et les incompatibilités d’emploi du temps sont des freins majeurs à la participation au DPC}

Dans les pays et les économies de l'OCDE participant à l'Enquête TALIS, près de deux enseignants sur cinq (38 \%) indiquent qu'il n'y a pas d'activités pertinentes de développement professionnel, ce qui entrave fortement leur participation au développement professionnel (voir le graphique 2). Cet écart entre l'offre en matière de DPC et les besoins réels peut avoir une incidence sur la participation générale aux activités s'y rattachant comme sur le nombre d'activités de DPC suivies.

À l'échelle des pays, il existe une corrélation négative modérée entre la participation à différentes activités de DPC et le sentiment d'absence d'activités pertinentes de développement professionnel (le coefficient de corrélation linéaire $r$ est équivalent à -.39 [r2 $=0,15]$ parmi les 30 pays de l'OCDE et équivalent à -.49 [r2 = 0,24] parmi les 44 pays de l'Enquête TALIS dont les données sont disponibles). Par exemple, c'est au Chili et au Portugal, là où le pourcentage d'enseignants indiquant un manque d'activités de développement professionnel pertinentes est le plus élevé (60 et $62 \%$ respectivement), que le nombre d'activités professionnelles différentes auxquelles les enseignants ont participé est le plus faible (moins de trois activités). En revanche, c'est au Kazakhstan et dans la Fédération de Russie, là où le pourcentage d'enseignants indiquant un manque d'activités de développement professionnel pertinentes est le plus faible (19 et $21 \%$ respectivement), que les enseignants indiquent qu'ils ont participé à plus de six activités différentes de développement professionnel. Cependant, il existe des exceptions à cette tendance générale : par exemple, en Norvège, tant le pourcentages d'enseignants participant à des activités de développement professionnel que le pourcentage signalant un nombre insuffisant d'activités pertinentes sont tous deux parmi les plus faibles des pays et des économies participant à l'Enquête. 


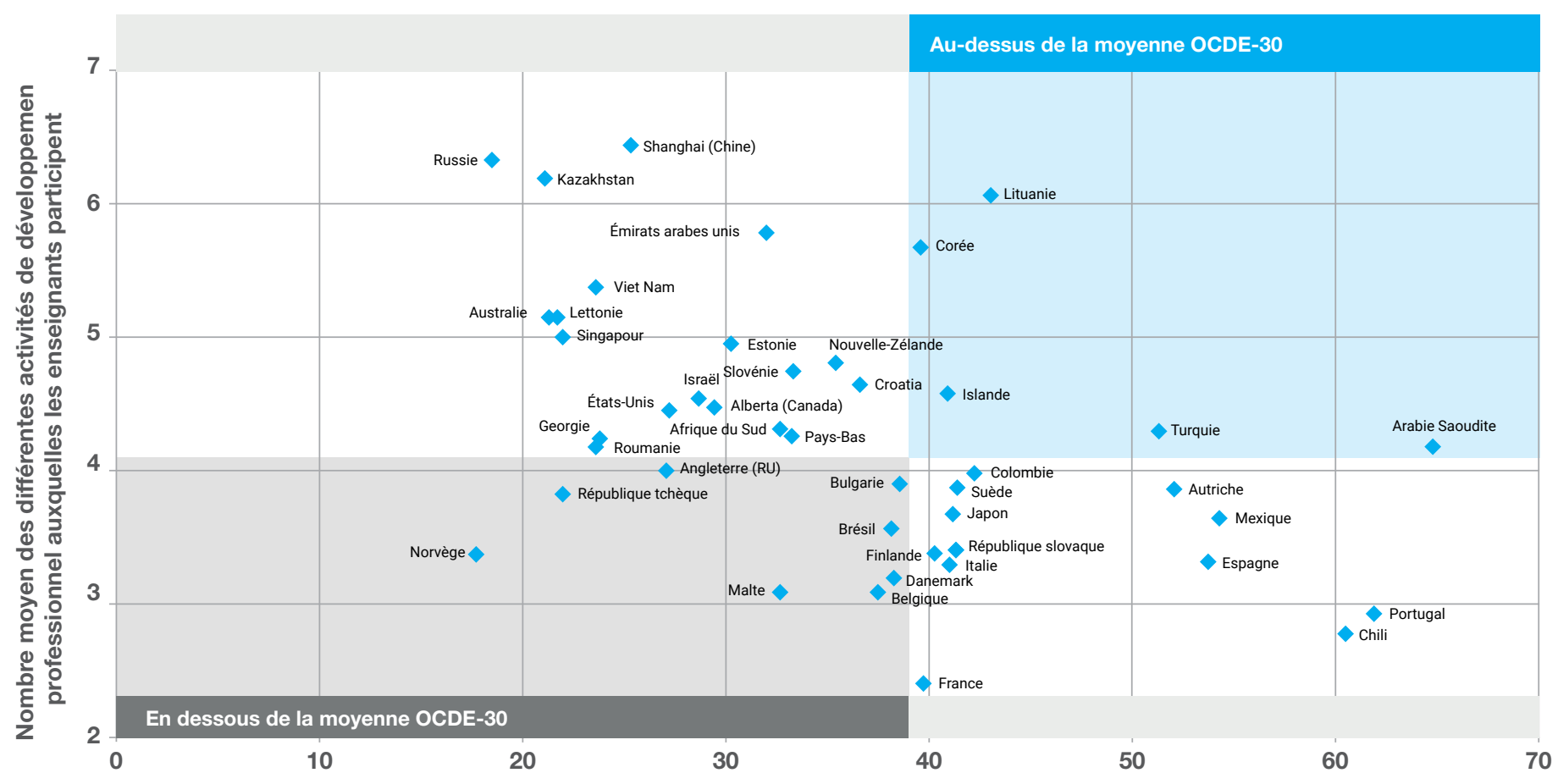

Pourcentage d'enseignants qui déclarent qu'aucun développement professionnel pertinent $n^{\prime}$ est $^{\text {offert }}{ }^{1}$

1. Ce graphique fait référence aux activités de développement professionnel auxquelles les enseignants ont participé au cours des douze mois précédant l'Enquête.

Remarque : Les pays et les économies figurent uniquement dans le graphique si les données disponibles étaient suffisantes concernant le nombre moyen d'activités différentes de développement professionnel auxquelles les enseignants ont pu participer et concernant le pourcentage d'enseignants ayant indiqué qu'il n'y avait pas suffisamment d'activités pertinentes de développement professionnel. La valeur moyenne des trente pays de l'OCDE couvre tous les pays de l'OCDE ayant participé à l'Enquête TALIS 2018, à l'exception de la Hongrie. Source : OCDE, Base de données TALIS 2018, tableaux I.5.7 et I.5.36.

La participation générale aux activités de DPC est faible dans les pays où le pourcentage d'enseignants indiquant un manque d'activités de développement professionnel pertinentes est très élevé. Par exemple, en Arabie saoudite, pays où le pourcentage d'enseignants indiquant un manque d'activités de développement professionnel pertinentes est le plus élevé, seuls $86 \%$ des enseignants avaient participé à 1 activité de DPC au cours de l'année écoulée, ce qui est bien inférieur à la moyenne de l'OCDE (94\%).

Le manque de temps est l'un des autres freins à la participation des enseignants aux activités de DPC. En moyenne, dans les pays et les économies de l'OCDE participant à l'Enquête TALIS, plus de la moitié des enseignants (54 \%) indiquent que le développement professionnel est incompatible avec leur emploi du temps professionnel. Les contraintes de temps constituent un obstacle majeur dans la plupart des pays et sont particulièrement importantes dans la CABA (Argentine), en Corée, au Japon et au Portugal, où plus de trois enseignants sur quatre indiquent qu'elles posent problème.

De plus, 48 \% des enseignants indiquent qu'il n'y pas d'incitation à participer au développement professionnel (alors que la moyenne de l'OCDE est équivalente à $32 \%$ ), ce qui est souligné par le manque de soutien des employeurs. En Arabie Saoudite, au Brésil, en Colombie, en Corée, au Mexique et au Portugal, plus de $65 \%$ des enseignants indiquent que leur employeur est particulièrement réticent à soutenir leur participation aux activités de DPC, tandis qu'en Australie, en Estonie, en Géorgie, en Islande, en Lettonie, en République slovaque et en République tchèque, seule une faible proportion des enseignants indiquent qu'ils manquent de soutien de la part de leur employeur (moins de $15 \%$ ).

\section{Les enseignants indiquent que leurs besoins en matière de développement professionnel sont importants dans certains domaines difficiles}

Les enseignants ont répondu qu'ils avaient un besoin important en matière de DPC dans le domaine de « 'enseignement aux élèves ayant des besoins spécifiques » (22 \%), " des compétences en TIC à l'appui de l'enseignement » (18 \%) et de « l'enseignement en milieu multiculturel ou plurilingue » (15 \%) (OCDE, Base de données TALIS 2018, tableau I.5.21). Ces besoins importants en matière de DPC en enseignement en milieu multiculturel ou plurilingue découlent de la diversité culturelle et ethnique croissante des salles de classe, tandis que les besoins liés à l'enseignement aux élèves ayant des besoins spécifiques sont liés à la nécessité d'améliorer l'équité dans l'éducation, en particulier dans les cadres inclusifs. 
Lintégration des technologies de l'information et de la communication (TIC) dans les salles de classe constitue également un défi important auquel les systèmes éducatifs font face à l'heure actuelle. D'après les enseignants, les besoins en matière de formation concernant les compétences en TIC pour l'enseignement varient entre $5 \%$ en Angleterre et $55 \%$ au Viet Nam.

Graphique 3. Besoins des enseignants en matière de développement professionnel ${ }^{1}$ de leurs compétences en TIC pour l'enseignement, en fonction de leur participation aux activités de développement professionnel

Pourcentage d'enseignants du premier cycle du secondaire qui ont répondu qu'ils avaient un besoin important en matière de développement professionnel de leurs compétences en $\mathrm{TIC}^{2}$ pour l'enseignement

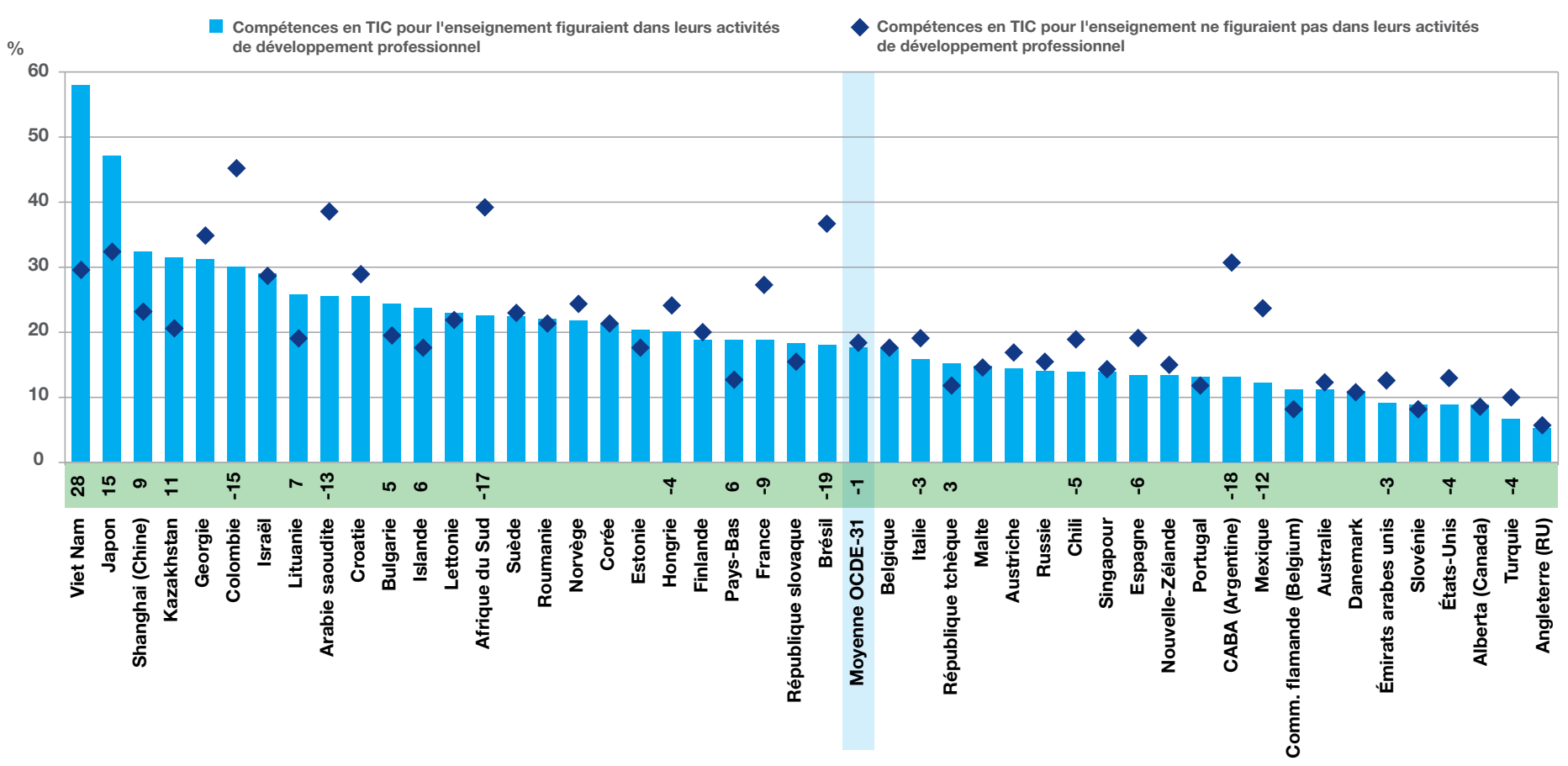

1. Ce graphique fait référence aux activités de développement professionnel auxquelles les enseignants ont participé au cours des douze mois précédant l'Enquête. 2. Technologies de l'information et de la communication (TIC).

Remarque : Les différences statistiquement importantes entre les enseignants qui ont inclus les compétences en TIC pour l'enseignement dans leurs activités de développement professionnel et ceux qui ne les ont pas inclues sont indiquées après le nom du pays ou de l'économie.

Source : OCDE, Base de données TALIS 2018, tableau 1.5.24.

L'utilisation la plus adaptée des TIC dans l'enseignement fait l'objet de débats intenses dans la communauté scientifique et les résultats montrent que les besoins restent élevés même après la participation à des activités de développement professionnel axées sur les compétences en TIC pour l'enseignement. Dans les pays et les économies de l'OCDE participant à l'Enquête TALIS, le pourcentage d'enseignants déclarant avoir un besoin élevé en matière de formation aux compétences en TIC pour l'enseignement après avoir suivi une activité de formation n'est inférieur que d'un seul point au pourcentage des enseignants signalant le même besoin mais sans avoir suivi de formation appropriée (voir le graphique 3). En Islande, au Japon, au Kazakhstan, en Lituanie, à Shanghai (Chine) et au Viet Nam, les besoins en matière de formation aux compétences en TIC pour l'enseignement atteignent cinq points de pourcentage de plus lorsque ces formations ont été suivies. Dans ces pays, les enseignants semblent vouloir suivre d'autres formations plus approfondies. L'inverse se vérifie en Afrique du Sud, en Arabie Saoudite, au Brésil, dans la CABA (Argentine), au Chili, en Colombie, en Espagne, en France et au Mexique, où les enseignants indiquent que leurs besoins en matière de formation sont plus faibles lorsqu'ils ont suivi ces formations (écart de plus de cinq points de pourcentage). 


\section{Pour conclure}

Le développement professionnel continu (DPC) est un aspect essentiel au professionnalisme des enseignants et a des répercussions sur les pratiques pédagogiques dans les salles de classe. De plus, l'efficacité personnelle et la satisfaction professionnelle des enseignants sont plus importantes lorsque les activités de développement professionnel ont des retombées positives sur leur travail.

Les enseignants ont répondu qu'ils avaient un besoin important en matière de DPC dans de nouveaux domaines qui sont souvent difficiles, tels que l'enseignement aux élèves ayant des besoins spécifiques, les compétences en TIC à l'appui de l'enseignement et l'enseignement en milieu multiculturel ou plurilingue. Cependant, différents obstacles entravent leur participation aux activités de DPC, tels que l'inadéquation entre le contenu de la formation et leurs besoins, les incompatibilités d'emploi du temps professionnel, l'absence d'incitations et l'absence de soutien des employeurs.

Pour donner aux enseignants la possibilité d'accéder aux activités de DPC et les rendre pertinentes, il est essentiel d'éliminer les obstacles à leur participation à ces activités en mettant en place des mesures incitatives et des structures qui les soutiennent, telles que des créneaux horaires qui leur sont réservés, en proposant des activités qui répondent aux besoins des enseignants. Les systèmes éducatifs pourraient également tirer profit de formes efficaces de développement professionnel, telles que les approches collaboratives et d'apprentissage actif, pour améliorer la qualité générale des activités de développement professionnel offertes au personnel enseignant. Ces efforts peuvent aider les enseignants à se former tout au long de leur vie et à évoluer dans leur profession.

\section{Consulter}

\section{www.oecd.org/education/talis/}

\section{Contacter}

Markus Schwabe (markus.schwabe@oecd.org)

Pablo Fraser (pablo.fraser@oecd.org)

\section{Pour en savoir plus}

OCDE (2019), Résultats de TALIS 2018 (volume I): Des enseignants et chefs d'établissements en formation à vie, TALIS, Éditions OCDE, Paris, https://doi.org/10.1787/5bb21b3a-fr. Ce rapport examine les connaissances et les compétences en lien avec le professionnalisme des enseignants et des chefs d'établissement.

OCDE (2020), Résultats de TALIS 2018 (volume II): Des enseignants et chefs d'établissement comme professionnels valorisés, TALIS, Éditions OCDE, Paris. Ce rapport sera publié début 2020 et portera sur le prestige de la profession, les opportunités professionnelles, la culture collaborative, ainsi que la responsabilité et l'autonomie professionnelles.

Les notes par pays de TALIS exposent les principales conclusions tirées de l'Enquête TALIS 2018 qui a été adaptée aux pays concernés dans une perspective internationale. www.oecd.org/education/talis/talis-2018-country-notes.htm.

Cet ouvrage est publié sous la responsabilité du Secrétaire général de l'OCDE. Les opinions et les interprétations exprimées ne reflètent pas nécessairement les vues de l'OCDE ou des gouvernements de ses pays membres.

Ce document et toute carte qu'il peut comprendre sont sans préjudice du statut de tout territoire, de la souveraineté s'exerçant sur ce dernier, du tracé des frontières et limites internationales, et du nom de tout territoire, ville ou région.

Les données statistiques concernant Israël sont fournies par et sous la responsabilité des autorités israéliennes compétentes. L'utilisation de ces données par l'OCDE est sans préjudice du statut des hauteurs du Golan, de Jérusalem-Est et des colonies de peuplement israéliennes en Cisjordanie aux termes du droit international.

La copie, le téléchargement ou l'impression du contenu OCDE pour une utilisation personnelle sont autorisés. Il est possible d'inclure des extraits de publications, de bases de données et de produits multimédia de l'OCDE dans des documents, présentations, blogs, sites internet et matériel pédagogique, sous réserve de faire mention de la source et du copyright. Toute demande en vue d'un usage public ou commercial ou concernant les droits de traduction devra être adressée à rights@oecd.org.

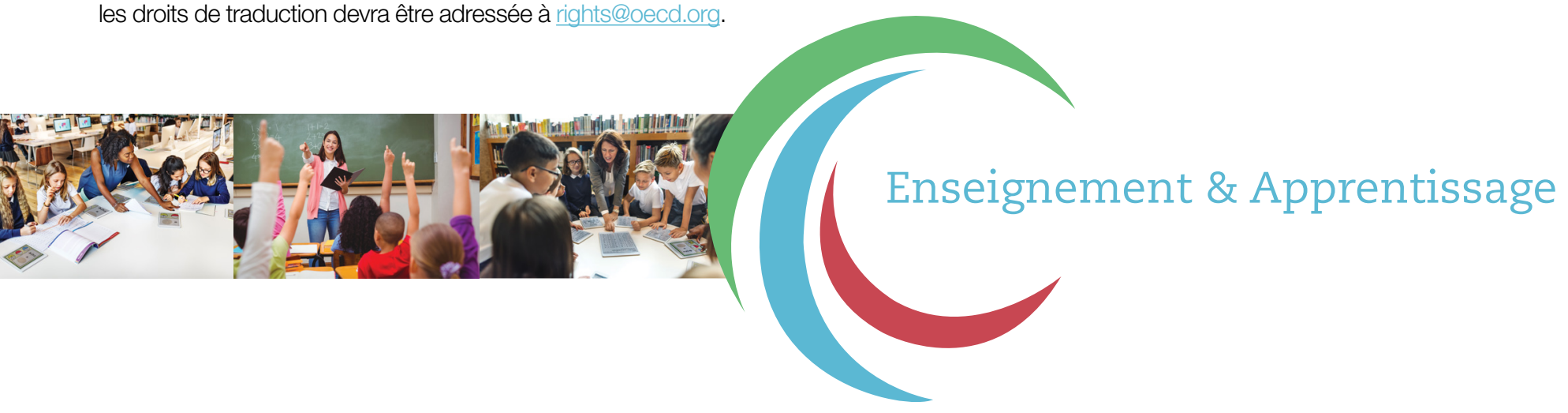

